\title{
El género Amanita Pers. eX HoOKer en la PROVINCIA DE LEÓN
}

\author{
Juan A. SÁNCHEZ RODRÍGUEZ, Jaime ANDRÉS RODRÍGUEZ, Oscar \\ GARCÍA PRIETO, Arsenio TERRÓN ALFONSO, Basilio LLAMAS FRADE, \\ Eduardo ARROJO MARTÍN, \& Tomás PÉREZ JARAUTA
}

RESUMEN: Se citan 23 taxa del género Amanita en la provincia de León (Noroeste de España). Se acompaña una clave para su determinación, su distribución provincial, ecología, así como su relación con las series de vegetación.

Palabras clave: Taxonomía, Amanita, Basidiomycetes, Noroeste de España.

SUMMARY: Twenty three taxa of genus Amanita in León province (NW Spain) are quoted. An identification key of all these taxa is provided, as well as their provincial distribution, ecology and their relation with the vegetation series.

Key words: Taxonomy, Amanita, Basidiomycetes, NW of Spain.

\section{INTRODUCCIÓN}

El género Amanita, fue siempre objeto de particular atención pues, no en vano, abarca desde las especies más apreciadas (A. caesarea) a las más tóxicas ( $A$. phalloides, A. verna, A. virosa, etc.).

En la provincia de León se encuentra abundantemente representado y se han recolectado 23 especies. Con ellas se ha confeccionado una clave para su determinación y, al mismo tiempo, sin que haya hecho un muestreo estadístico (y sólo a título orientativo) se han enfrentado las especies recogidas con las series de vegetación existentes en el territorio ( Tabla I ). También se aporta en esta ocasión un mapa de distribución provincial del material estudiado en cuadrículas U.T.M. de 10 x $10 \mathrm{Km}$. para cada especie. 


\section{CATÁLOGO}

Amanita aspera (Vitt.) Quél.

Distribución: Localizada en Villamizar ( 30TUN21), La Bañeza (30TTM38) y Pinilla de la Valdería (29TQG47), pertenecientes a la Región Mediterránea.

Ecología: Frecuente en melojares, castañares y encinares con ombroclima húmedo a subhúmedo y sustrato ácido.

Amanita caesarea (Scop. ex Fr.) Pers. ex Schw.

Distribución: Localizada en Vidanes (30TUN23),Sta. María del Condado (30TUNO3),Montejos (30TTN71), Jiménez de Jamuz (30TTM58), Pinilla de la Valdería (29TQG47) y S. Justo de La Vega (29TQH30), pertenecientes a la Región Mediterránea.

Ecología: Especie mediterránea, relativamente frecuente algunos años a finales de verano, que fructifica en melojares, castañares y encinares con ombroclima húmedo a seco y sustrato indiferente.

Amanita citrina (Schuff.) S.F. Gray

Distribución: Localizada en Camposagrado (30TTN73), Santibáñez de la Isla (30TTN50), Monte S. Isidro (30TTN81) y Riego de Ambrós (29TQH01); todos ellos perteneciente a la Región Mediterránea

Ecología: Frecuente en castañares, pinares y encinares con ombroclima húmedo a subhúmedo y sustrato ácido.

Amanita citrina var alba (Vitt.) Gilbert

Distribución: Localizada en Santibáñez de la Isla (30TTN50) perteneciente a la Región Mediterránea.

Ecología: Bastante rara, la hemos visto en encinares subhúmedos con sustratos ácidos.

Amanita crocea (Quél.) Sing.

Distribución: Localizada en La Seca (30TTN83), Ardoncino (30TTN80),La Bañeza (30TTM68) y Villamizar(30TUN21) pertenecientes al Sector Leonés de la Región Mediterránea.

Ecología: Frecuente en melojares y encinares con ombroclima húmedo a seco y sustrato ácido.

\section{Amanita curtipes Gilbert}

Distribución: Localizada en Ardoncino (30TTN80), Monte del Duque (30TTM96), Santibáñez de La Isla (30TTN50), Herreros de Jamuz (30TTM58); pertenecientes a la Región Mediterránea.

Ecología: Especie mediterránea, frecuente en melojares y encinares con ombroclima húmedo a seco y en sustrato indiferente. Generalmente primaveral.

Amanita echinocephala Vitt.

Distribución: Localizada en Ardoncino (30TTN80), Ferral del Bernesga (30TTN82), Santovenia de La Valdoncina (30TTN81), Pobladura de Pelayo García (30TTM78); perteneciente al Sector Leonés de La Región Mediterránea.

Ecología: Poco frecuente, la hemos visto en melojares y encinares con ombroclima húmedo a seco y también en praderas. 


\begin{tabular}{|c|c|c|c|c|c|c|c|c|c|c|c|c|c|c|c|c|c|c|c|}
\hline Dsนวอxว & & * & & & - & & & & & & & & & & & & & & \multirow{28}{*}{ 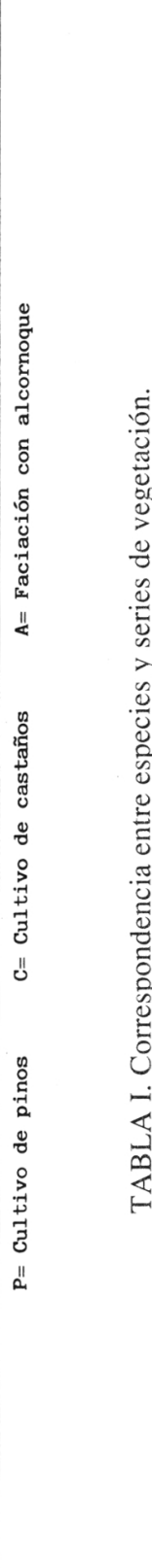 } \\
\hline pss?ds & & & & * & & & & & & & 0 & & & & & & & & \\
\hline suวəsaqne & & $\stackrel{*}{*}$ & & * & * & & * & & & . & ○ & a & $*$ & & & & & & \\
\hline pqufiyduod & & 赵 & & & & & & & & & & & & * & & & & & \\
\hline sap?oz2pyd • & & * & & & & & & & & & * & * & * & * & * & & & & \\
\hline ołnąou?uqum & & & & & & $*$ & * & & & & & $*$ & & & & & & & \\
\hline paqum $2 d \cdot$ xen pqDu?bon & & & & & & & & $*$ & & & & & & & & & * & * & \\
\hline р7Du?bon - & & & & & & & $*$ & & & & & * & $*$ & * & & & & & \\
\hline pu?uazupd & & & & & & & * & & & * & 0 & $\begin{array}{l}0 \\
a\end{array}$ & & $*$ & & & & & \\
\hline 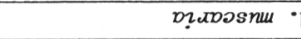 & & $a^{*}$ & & & & & $a$ & & & & o. & $\begin{array}{ll}u \\
* \\
*\end{array}$ & $a$ & . & & & & & \\
\hline 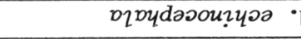 & & & & & & & & & & & & * & & * & & & * & & \\
\hline ?2u?po77?n & & & & & & & & & & & & . & & & & & * & & \\
\hline s?muxof? 20 qoups & & & & & & & & & & & & a & $*$ & * & * & & & & \\
\hline Duadso & & & & & & & & & & & $\begin{array}{ll}* \\
\omega \\
\end{array}$ & $*$ & * & & & & & & \\
\hline Dap?ono & & & & & & & & & & & & 0 & & * & * & & & & \\
\hline sad?zano & & & & & & & & & & & * & 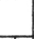 & * & * & * & & & & \\
\hline $27 d \partial q 2.6 \cdot$ & & & & & & & & & & & & $\begin{array}{l}* \\
a \\
\end{array}$ & & & & & & & \\
\hline Dqlo $\cdot$ xen Du?d7? & & & & & & & & & & & & . & * & & & & & & \\
\hline 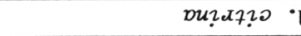 & & & & & & & & & & & 0 & $a$ & * & & & & & & \\
\hline Dutar $\cdot$ & & & & & & & & & & & & & * & & * & & & & \\
\hline Dq1D - xes sap?ozzoyd & & & & & & & & & & & & & $*$ & * & & & & & \\
\hline paznf & & & & & & & & & & & * & & * & * & * & & & & \\
\hline Dasous $\cdot$ & & & & & & & & & & & & * & & * & & & & & \\
\hline 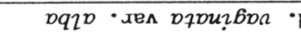 & & & & & & & & & & & & & & & & & & & \\
\hline D7Dxnou? & & & & & & & & & & & $\begin{array}{l}2 \\
0 \\
0\end{array}$ & $*$ & $\begin{array}{ll}* \\
a\end{array}$ & * & & & & $*$ & \\
\hline р7риа6 $\cdot$ & & & & & & & & & & & $\begin{array}{ll}0 \\
0 \\
0\end{array}$ & 0 & $\begin{array}{ll}* \\
a\end{array}$ & & * & & & & \\
\hline Dadosano & & & & & & & & & & & 茪 & $*$ & * & & * & & & & \\
\hline 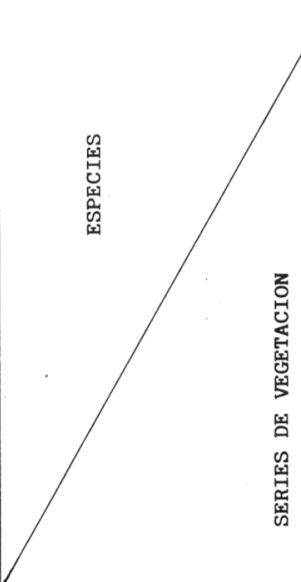 & & $\begin{array}{l}0 \\
0 \\
0\end{array}$ & & 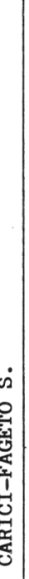 & 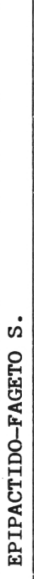 & 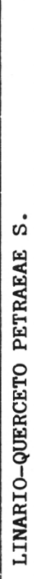 & 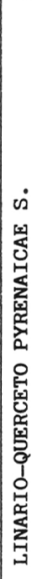 & $\begin{array}{l}0 \\
0\end{array}$ & & & 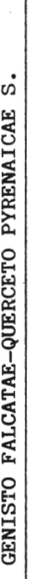 & 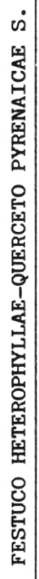 & 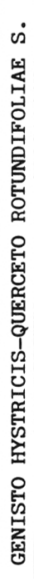 & $\begin{array}{l}\text { i } \\
\\
\end{array}$ & o & & 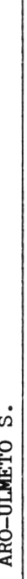 & 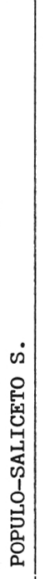 & \\
\hline
\end{tabular}


Amanita excelsa (Fr.) Kummer

Distribución: Localizada en Riosol (30TUN36); perteneciente a la Región Eurosiberiana.

Ecología: Especie rara, que la hemos visto en hayedos, con ombroclima hiperhúmedo y sustrato ácido.

Amanita fulva Schoff.

Distribución: Localizado en Jiménez de Jamuz (30TTM58), Monte del Duque (30TTM96), Ardoncino (30TTN80) y Corporales (29TQG97); pertenecientes a la Región Mediterránea.

Ecología: Frecuente en melojares y encinares con ombroclima húmedo a seco y sustrato indiferente.

Amanita gemmata (Fr.) Gilb.

Distribución: Localizada en Sancedo (29TPH92), Matachana (29TQH12), Santibáñez de La Isla (30TTN50), La Bañeza (30TTM68), Lorenzana (30TTN82) y Castrocalbón (30TTM47); pertenecientes a la Región Mediterránea

Ecología: Muy frecuente en pinares,castañares y encinares con ombroclima húmedo a subhúmedo y sustrato ácido. También observada en encinares con ombroclima seco y sustrato básico.

Amanita gilberti Beauseigneur

Distribución: Localizada en Lorenzana (30TTN82), perteneciente a la Región Mediterránea.

Ecología: Especie rara, la hemos visto en pinares y melojares con ombroclima húmedo y sustrato ácido.

\section{Amanita inaurata Secr.}

Distribución: Localizada en Sancedo (29TPH91), Matachana (29TQH12), La Seca (30TTN83) y Ardoncino (30TTN80), pertenecientes a la Región Mediterránea.

Ecología: Frecuente en pinares, castañares, melojares y encinares con ombroclima de seco a húmedo y sustrato ácido.

\section{Amanita muscaria (L. ex Fr.) Hooker}

Distribución: Localizada en Puebla de Lillo (30TUN17), La Uña (30TUN27), Rioscuro (29TQH25), Cuadros (30TTN83), Lorenzana(30TTN82), Camposagrado (30TTN73), La Robla (30TTN84), Corporales (29TQG07), Riego de Ambrós (29TQH01) y Sancedo (29TPH91), pertenecientes a la Región Eurosiberiana y Mediterránea.

Ecología: Muy frecuente en pinares, castañares, hayedos, abedulares y melojares con ombroclima subhúmedo a hiperhúmedo y sustrato ácido.

\section{Amanita ovoidea (Bull. ex Fr.) Quél}

Distribución: Localizada en Matachana (29TQH12), Alija del Infantado (30TTM66) y Monte del Duque (30TTM96), pertenecientes a la Región Mediterránea.

Ecología: Especie mediterránea, poco frecuente, la hemos visto en castañares y encinares con ombroclima de seco a húmedo y sustrato indiferente. 
Amanita pantherina (DC. ex Fr.) Secr.

Distribución: Localizada en Candín (29TPH84), Lorenzana (30TTN82), Santa María del Condado (30TUN03), Compludo (29TQH00) y Ardoncino (30TTN80), pertenecientes a la Región Mediterránea.

Ecología: Frecuente en melojares, castañares, pinares y encinares de ombroclima seco a húmedo y sustrato ácido.

Amanita phalloides (Vaill. ex Fr.) Secr.

Distribución: Localizada en Vegabaño (30TUN38), Santibáñez de la Isla (30TTN50), Villamizar (30TUN21), Lorenzana (30TTN82), Molinaseca (29TQH01), Ardoncino (30TTN80), La Bañeza (30TTM68), Jiménez de Jamuz (30TTM58), Venta de la TuertaCuadros (30TTN83) y León (30TTN81), pertenecientes a la Región Eurosiberiana y Mediterránea.

Ecología: Frecuente en hayedos, melojares, pinares, castañares y encinares con ombroclima seco a hiperhúmedo y sustrato indiferente.

Amanita phalloides var. alba (Vitt.) Gilb.

Distribución: Localizada en Pombriego (29TPG89), La Bañeza(30TTM68) y Santibáñez de la Isla (30TTN50), pertenecientes a la Región Mediterránea.

Ecología: Poco frecuente, la hemos visto en encinares y alcornocales con ombroclima seco a subhúmedo y sustrato ácido.

Amanita porphyria (A. \& S. ex Fr.) Secr.

Distribución: Localizada en Puebla de Lillo (30TUN17) y Villomar (30TUN21), pertenecientes a la Región Eurosiberiana y Mediterránea respectivamente.

Ecología: Poco frecuente, la hemos visto en pinares con ombroclima hiperhúmedo y sustrato ácido, y en encinares con ombroclima seco y sustrato ácido.

Amanita rubescens (Pers. ex Fr.) Gray

Distribución: Localizada en Burón (30TUN36), Puerto de Panderrueda (30TUN37), Puebla de Lillo (30TUN17), Llombera (30TTN84), Rioscuro (29TQH25), Truchillas y Corporales (29TQG07), Cabañas de la Dormilla (29TPH92), Ocero (29TPH93), Candín (29TPH84), La Bañeza (30TTM68), Santibáñez de la Isla (30TTN50) y Camposagrado (30TTN73), pertenecientes a la Región Eurosiberiana y Mediterránea.

Ecología: Muy frecuente en hayedos, pinares, castañares, melojares, abedulares y encinares, con ombroclima subhúmedo a hiperhúmedo y sustrato indiferente.

Amanita spissa (Fr.) Kummer

Distribución: Localizada en Burón (30TUN36) y Castrocalbón (30TTM47), pertenecientes a la Región Eurosiberiana y Mediterránea respectivamente.

Ecología: Poco frecuente, la hemos visto en hayedos y castañares con ombroclima húmedo a hiperhúmedo y sustrato indiferente.

Amanita strobiliformis (Vitt.) Quél.

Distribución: Localizada en Lorenzana (30TTN82), Monte de San Isidro-León (30TTN81), Santibáñez de la Isla (30TTN50), Monte del Duque (30TTM96) y Alija del Infantado (30TTM66), pertenecientes a la Región Mediterránea. 
Ecología: Frecuente, fructifica en pinares y encinares con ombroclima seco a subhúmedo e indiferente al sustrato.

\section{Amanita umbrinolutea Secr.}

Distribución: Localizada en Balouta (29TPH75), Rioscuro (29TQH25), Fasgar (29TQH24) y Rioseco de Tapia (30TTN73), pertenecientes a la Región Eurosiberiana y Mediterránea.

Ecología: Frecuente en abedulares y melojares con ombroclima húmedo-hiperhúmedo y sustrato ácido.

Amanita vaginata (Bull. ex Fr.) Vitt.

Distribución: Localizada en Fasgar (29TQH24), Geras de Gordón (30TTN75), Valsemana (30TTN83), Rioseco de Tapia (30TTN73), Santibáñez de la Isla (30TTN50) y Ardoncino (30TTN80), pertenecientes a la Región Eurosiberiana y Mediterránea.

Ecología: Frecuente en abedulares, melojares y encinares con ombroclima seco a húmedo y sustrato ácido.

Amanita vaginata var. alba Gilb.

Distribución: Localizada en Valsemana (30TTN83), perteneciente a la Región Mediterránea.

Ecología: Poco frecuente, la hemos visto en una chopera sobre sustrato básico.

Amanita vaginata var. plumbea Schaff.

Distribución: Localizada en Cabornera (30TTN75), Valsemana y Cascantes (30TTN83) y Lorenzana (30TTN82), pertenecientes a la Región Eurosiberiana y Mediterránea.

Ecología: Frecuente en bosques y prados de ribera (fresnedas, olmedas, saucedas, choperas, etc.) e indiferente al sustrato.

Amanita verna (Bull. ex Fr.) Pers. ex Vitt.

Distribución: Localizada en Santalavilla (29TPG99), Pombriego (29TPG89), Valdelaguna (30TUM39) y Santibáñez de la Isla (30TTN50), pertenecientes a la Región Mediterránea.

Ecología: Poco frecuente, la hemos visto en encinares y alcornocales con ombroclima seco a subhúmedo e indiferente al sustrato. Preferentemente primaveral.

Amanita vittadini (Mor.) Vitt.

Distribución: Localizada en el Campus Universitario de León (30TTN81), perteneciente e la Región Mediterránea.

Ecología: Especie mediterránea, poco frecuente, solo la hemos visto en una pradera de sustrato básico y de dominio climácico de olmeda.

\section{CLAVE}

1 -Esporas no amiloides. Margen del sombrero con estrías

1' -Esporas amiloides. Margen del sombrero sin estrías, liso 
2 -Pie sin anillo (Subgenero. Vaginaria o Amanitopsis) ....................................................4

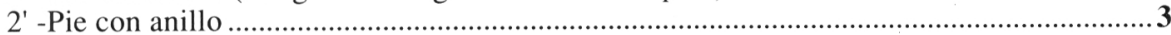

3 - Volva membranosa, ersistente, no bulbosa, (Subgénero Amanita). Anillo persistente, amarillo, sombrero carnoso anaranjado y láminas amarillas ........................... . caesarea

3' -Volva en grumos, floconosa (Subgénero Amanitaria) ...................................................10

4 - Especies con volva floconosa, friable, no envolvente y lacerada. Sombrero pardo grisáceo o amarillo marrón con numerosas placas grises ............................................. A. inaurata

4' - Especies con volva membranosa, envolvente y persistente ............................................5

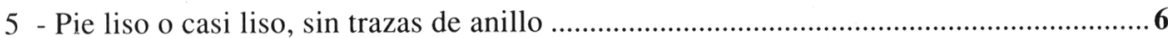

$5^{\prime}$ - Pie floconoso o atigrado, con evidentes trazas de anillo en la juventud ........................9

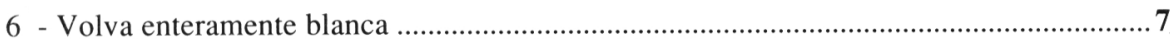

6 ' - Volva blanca pero con la orla de otro color ................................................................ 8

7 - Sombrero gris ceniza o color carne

A. vaginata

7' - Sombrero enteramente blanco al igual que el pie y volva .............. A. vaginata var. alba

8. - Sombrero leonado rojizo. Pie más claro. Volva blanca orlada de rojizo ............. f fulva $8^{\prime}$ - Sombrero gris-plomo. Pie blanco con flocosidades grisáceas. Volva blanco crema ........ A. vaginata var. plumbea

9 - Sombrero amarillo rojizo, pie con escamosidades del mismo color en zigzag...A. crocea 9'- Sombrero gris pardo oliva, zonado. Pie jaspeado de pardo A. umbrinolutea

10 - Sombrero rojo anaranjado, cubierto de placas blancas algodonosas piramidales. Volva fragmentada por escamas

$10^{\prime}$ - Sombrero no rosa anaranjado 11

11 - Sombrero marrón grisáceo, con placas harinosas, piramidales, blancas. Volva circuncisa no escamosa

A. pantherina

11 ' - Sombrero amarillo ocráceo o verde con o sin escamas blancas. Volva circuncisa, grumosa, algo napiforme

A. gemmata

12 - Especies con volva membranosa, persistente .................................................................. 13

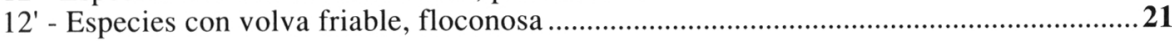

13 - Pie con anillo fugaz, algodonoso. Sombrero blanco con tendencia a oscurecer (Subgénero Amidella)

13' - Pie con anillo y volva persistente (Subgénero Amanitina)

14 - Especies con volva membranosa sólo en la base y friable en el ápice. Sombrero amarillocitrino, blanco o pardo oliváceo con placas harinosas (Sección mappae)

14'- Especies con volva membranosa en gran parte del pie. Sin placas harinosas (Sección phalloidae)

15 - La carne enrojece al corte. Volva en forma de saco

A. curtipes

15 ' - La carne permanece inmutable (no enrojece)

16 - Pie muy enterrado, algo napiforme. Volva rodeada de un collar. Sombrero de 6-10 cm. blanco 
16 - Pie floconoso. Volva muy grande, tenaz. Sombrero robusto de has ta $20 \mathrm{~cm}$., blanquecino A. ovoidea

17 - Sombrero de pardo púrpura a violáceo con placas harinosas grisáceas A. porphyria

$17^{\prime}$ - Sombrero amarillo citrino o blanco

18 - Sombrero amarillo citrino con placas ocráceas. Bulbo voluminoso redondo. Olor a patata

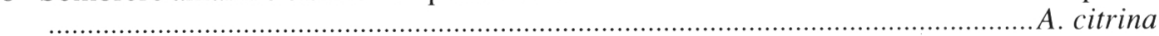

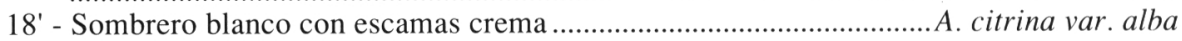

19 - Sombrero verdoso amarillento. Pie blanco con zonaduras verde pálido. Volva blanca e interior verdoso ...

A. phalloides

19' - Sombrero blanco 20

20 - Sombrero casi completamente blanco con esfumaciones verdosas. No amarillea a la potasa y a la sosa A. phalloides var. verna

$20^{\prime}$ - Sombrero blanco sucio, el centro ligeramente rosado. Sí amarillea con la potasa y la sosa ..A. verna

21 - Anillo membranoso, amplio y persistente. Esporas elípticas. Sombrero con placas harinosas (Subgénero Amplariella).

21' - Anillo cremoso, fugaz, en grumos. Esporas elípticas pero más o menos alargadas. Sombrero con verrugas piramidales (Subgénero Aspidella)

22 - La carne cambia de color al corte, enrojece sobre todo en la base del pie. Sombrero pardo rojizo vinoso con escamas blanco-grisáceas A. rubescens

22' - La carne es inmutable, no enrojece 23

23 - Anillo decorado de amarillo en el borde. Sombrero marrón grisáceo con placas amarillas

23' - Anillo blanco, estriado, sombrero gris pardo o marrón claro . A. aspera

24 - Talla del pie mediana de 10-15 cm., no muy enterrado, algo bulboso y decorado

24 ' - Talla del pie grande, de $15-20 \mathrm{~cm}$., muy enterrado, no bulboso. A. excelsa

25 - Sombrero con escamas no muy abultadas grises o blanco-cereo. Pie sin escamas.... A. strobiliformis

25 - Sombrero con escamas abultadas. Pie con escamas más o menos marcadas . .26

26 - Sombrero blanco con escamas o verrugas piramidales anchas, robustas y muy marcadas. Pie con escamas igualmente marcadas A. vittadinii

26 - Sombrero blanco argenteo o marfil con escamas no tan abultadas, piramidales. Pie con escamas tenues sólo en torno a la base y volva A. echinocephala

\section{COMENTARIOS A LA TABLA}

- Cualitativamente es más rica la Región Mediterránea que la Eurosiberiana.

- Cualitativamente son más ricas las series de vegetación climatófilas que las series edafo-higrófilas, independientemente de la Región biogeográfica en que se encuentren. 
- Las series que llevan como cabecera Quercus rotundifolia y Quercus pyrenaica son las más ricas en especies de Amanita.

- Se aprecia una preferencia por los cultivos de pinos por parte de A. muscaria, y en menor grado de $A$. gemmata, A. inaurata, A. citrina y A. rubescens, del mismo modo que se aprecia una preferencia por los cultivos de castaños por parte de $A$. aspera y A. pantherina.

- La máxima diversidad de especies se aprecia en los encinares del Genisto hystricis-Querceto rotundifoliae sigmetum y del Festuco heterophyllae-Querceto pyrenaicae sigmetum, pudiendo establecerse como territorio más favorable para la presencia de un mayor número de especies del género Amanita los sectores biogeográficos Orensano-Sanabriense, Leonés, y en menor cuantía el sector CastellanoDuriense.

- Debe reseñarse también la aparente exclusividad de A. excelsa por la serie del Luzulo-Fagetum sigmetum, así como de A. vaginata var. alba por la serie del PopuloSaliceto sigmetum, la de A. vittadini por la serie Aro-Ulmetum sigmetum, y la de A. citrina var. alba y A. phalloides var. alba por la serie del Genisto hystricis-Querceto rotundifoliae sigmetum.

\section{APUNTES SOBRE LA BIOGEOGRAFÍA DE LA PROVINCIA DE LEÓN}

La provincia de León en base a lo señalado por diversos autores (DIAZ GONZALEZ \& PENAS, 1984) se divide en las siguientes unidades fitogeográficas:

Región Eurosiberiana

Provincia Orocantábrica

Sector Campurriano-Carrionés (1)

Sector Ubiñense-Picoeuropeano (2)

Sector Laciano-Ancarense (3)

Región Mediterránea

Provincia Castellano-Maestrazgo-

Manchega

Sector Castellano-Duriense (4)

Provincia Carpetano-Ibérico-Leonesa

Sector Orensano-Sanabriense (5)

Sector Leonés (6)

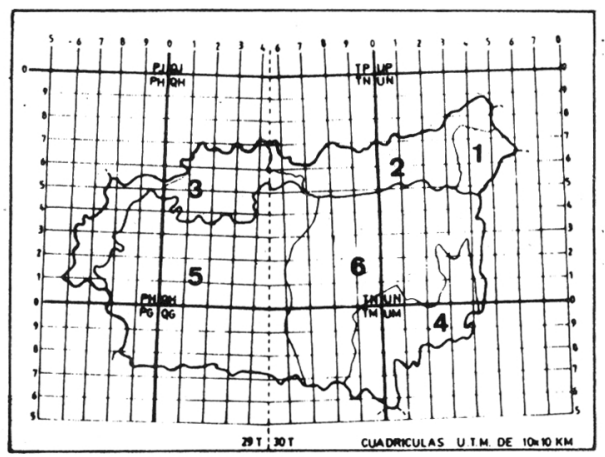

Mapa fitogeográfico de la provincia de León

\section{BIBLIOGRAFÍA}

GILBERT, E.J. -1940- Iconografia Mycologica. Vol 27. Supplementum I. Amanitaceae. Reimpresión de M. Canduso. Saronno, 1982 
MERLO, E.G. \& M. TRAVERSO -1983- I fungi. Le Amanite. Sagep Editrice. Genova

MESPLEDE, B.V. -1980- Le genre Amanita. Bull Soc. Mycol. France, 96(1): 8-47

MOSSER, M. -1980- Guida alla determinazione dei fungi (Polyporales, Boletales, Agaricales, Russulales)*. Saturnia. Trento

DÍAZ GONZÁLEZ, T.E. \& A. PENAS MERINO -1984- Bases para el mapa fitogeográfico de la provincia de León. Diputación Provincial de León.

RIVAS-MARTínEZ, S. -1987- Memoria del mapa de series de vegetación de España. Ministerio de Agricultura, Pesca y Alimentación. ICONA.

(Aceptado para su publicación en Junio de 1.990 )

Dirección de los autores: Departamento de Biología Vegetal. Facultad de Biología. Universidad de León. 